\title{
System for Automatic Camera Calibration Robust Against Blur and Lighting Conditions Changes
}

\author{
Yulian Pastarmov
}

\begin{abstract}
This paper presents a system for precise intrinsic and extrinsic camera calibration for monocular and stereo cameras. The presented approach is based on some well established research in the field and utilizes calibration patterns. The novelty of the presented method is the automatic detection of motion blur, that is common to the process of calibration based on moving the camera or the calibration object, especially under bad lighting conditions. The method allows for sub-pixel re-projection precision for cameras with perspective lenses and can be extended to omnidirectional cameras as well.
\end{abstract}

Key words: Camera Calibration, Intrinsic Camera Models, Monocular and Stereo Computer Vision.

\section{INTRODUCTION}

Many computer vision problems have straightforward solutions with the methods of linear algebra and analytic geometry. The problem usually arises from the fact that these algorithms never get applied to ideal images. Unless the images are software generated, but instead taken with real cameras, they are far from perfect representation of the world they capture. Various distortions are common in the images taken with a capturing device. The sources of these distortions lie in the physical capabilities of the hardware. For example, they are caused by the quality of the optics, the alignment of the camera elements, and the limitations of the sensors and electronics used by the manufacturers.

Naturally, the exact physical configuration of a camera is too complex to be represented $100 \%$ accurately by a computer model. Therefore, objectives lenses with a field of view of 100 degrees or less are represented by the so-called perspective or pinhole model, which is a simplification that still accurately matches the physical properties of the device. It's invention dates back to ancient times when people observed that light passing through a microscopic hole to a close projection surface accurately represents the scene in-front of the hole. This setup is called "Camera obscura". In modern times, the hole is substituted by a set of lenses and a mechanical iris that concentrates light rays on the surface of a light-sensitive sensor chip.

The imprecision of the optics and sensor positioning leads to systematic distortions which can be corrected if their parametrization is known. The relative positioning of the optical systems in stereo vision is also critical for the use of these systems and can be precisely computed. The computation of the camera parametrization usually happens in a structured way, eliminating one unknown after the other. However, since all parameters influence each other, this process is often iterative and preformed until convergence or some other exit criteria is reached.

This paper presents a method to estimate the optical projection and distortion parameters of the camera, known as intrinsic calibration, as well the relative positioning of the cameras in stereo and multi-view systems, known as extrinsic camera calibration. The proposed solution is based on the seminal paper by Zhang[6] but improves on it using ideas from the work of Devernay and Faugeras[7]. It also presents an automatic way of calibration pattern recognition similar to AprilCal[1] but augments this with motion blur recognition based on ideas from Crété-Roffet at al. [2].

\footnotetext{
Permission to make digital or hard copies of all or part of this work for personal or classroom use is granted without fee provided that copies are not made or distributed for profit or commercial advantage and that copies bear this notice and the full citation on the first page. Copyrights for components of this work owned by others than the author(s) must be honored. Abstracting with credit is permitted. To copy otherwise, or republish, to post on servers or to redistribute to lists, requires prior specific permission and/or a fee. Request permissions from Permissions@acm.org.

CompSysTech'16, 23-24 June 2016, Palermo, Italy

Copyright is held by the owner/author(s). Publication rights licensed to ACM. ISBN 978-1-4503-4182-0/16/06 ...\$15.00

http://dx.doi.org/10.1145/2983468.2983522
} 


\section{RELATED WORK}

In [6], Zhang studies the problem of calibrating a camera in depth and suggests a method to extract the intrinsic camera configuration from a set of images observing a known scene from different angles. Using the known correspondences between the observed points in the scene, one can build a linear system whose solution is the camera calibration matrix. He then proceeds to estimate the distortion coefficients of the optical system and finally optimizes the whole set of parameters in one large optimization problem. In [7], Devernay and Faugeras observe that proper treatment of the distortion parameters is crucial to the correct initialization of the intrinsics of the camera and suggest to first come up with a good estimate of the distortion so that it can be corrected before the general framework from [6] is applied. In order to do this, they study how straight lines are projected through optical systems with varying distortion and use those to estimate the distortion parameters. Lastly, these papers rely on the ability to detect some objects in the observed scene with known geometry. This is usually done by introducing a known planar surface with a printed pattern that can be distinguished from the background and has known geometry. Common patterns are a chessboard pattern or a circles pattern (see Fig.1) as used by OpenCV[8], Matlab's Calibration Toolbox[9] and others.
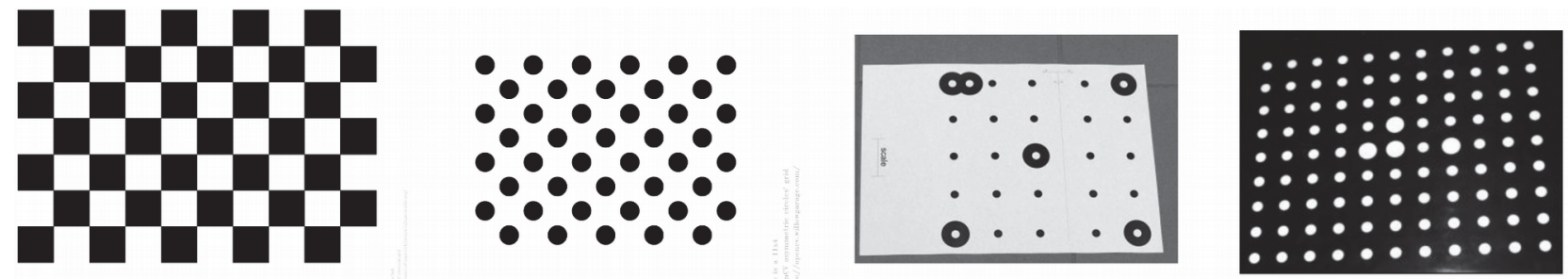

Fig 1: Various Calibration Patterns

AprilCal uses square fiducials (also known as markers) of different size with known identification which present a reliable way of verifying the detection and orientation of the pattern. The actual calibration process usually consists of the user taking multiple still images with the camera and feeding those through the calibration algorithm. Eventually, there is enough data to solve the equations and minimize the residual error of the optimization problem in which case the calibration is stored and the process ends. The process of taking images can be delegated to a robotised mechanism that moves the camera in certain trajectory and triggers the image acquisition process. Such a setup is costly and therefore seldom used in practice. This is also the motivation behind the need to introduce a measure for quality of the taken images, as bad images can significantly reduce the quality of the results or increase the convergence time of the process. The proposed method is based on a selection of blur metrics presented in [3] as well as another blur metric which does not require prior knowledge of image quality in the absence of noise presented in [2].

\section{PROPOSED METHOD}

\section{A. CAMERA PARAMETRIZATION}

As stated in the introduction, a completely accurate physical model of a camera is not feasible. Instead, for cameras with relatively narrow field of view, a simplification called the 'pinhole camera model' is applied. This model assumes that the lens system in front of the camera is capable of perfectly focusing the incoming light in a point in front of the light sensor of the camera and every incoming ray passing through this focal point does not change direction. Further model simplification is the fact that, instead of the mirrored image that would normally appear on a physical screen behind the focal point of the camera, a 'ghost' image plane is assumed to be in-front of the focal point at the same distance along optical axis as the real image plane which is a direct, non-mirrored 
representation of the observed scene. Here the optical axis denotes the line that passes through the centre of the lenses and is parallel to the image plane (see Fig.2).

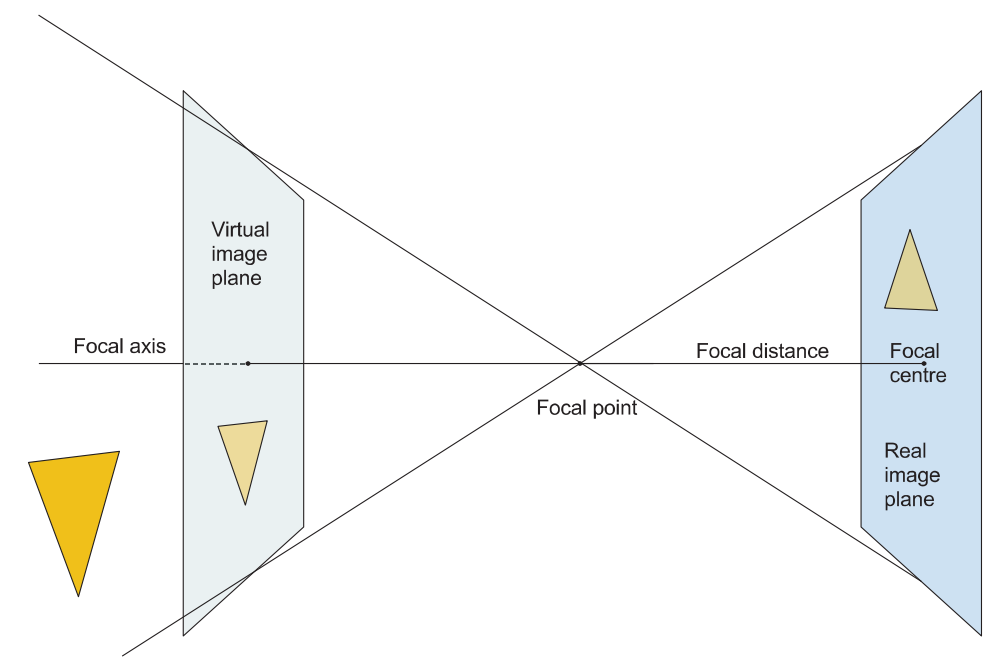

Fig 2: Pinhole Camera Model

This parametrization allows expressing the projection of the scene on the image plane as an affine transformation in $\mathrm{R}^{3}$ with the following matrix:

$$
K=\left|\begin{array}{ccc}
f_{x} & s & c_{x} \\
0 & f_{y} & c_{y} \\
0 & 0 & 1
\end{array}\right|
$$

In this matrix $f_{x}$ and $f_{y}$ denote the focal distance and usually are equal. The only reason they are denoted as 2 separate entities is to allow for non square image pixels in which case the focal distance expressed in pixels differs if taking the width or the height of the pixel. The parameter $s$ is called the skew factor and is usually set to 0 . It expresses the skewness of the image sensor which for most cameras is negligible. Lastly $c_{x}$ and $c_{y}$ represent the focal centre in the image plane in pixels. $K$ then projects rays onto the image plane by using the following equation:

$$
\left(\begin{array}{l}
x \\
y \\
z
\end{array}\right)=K\left(\begin{array}{l}
X \\
Y \\
Z
\end{array}\right)
$$

Where the vector $P=(X, Y, Z)^{T}$ denotes a point in space and $p=(x, y, z)^{T}$ denotes the de-normalized projection onto the image plane. In order to get a point on the actual image plane, $p$ is normalized by dividing by $z$, thus getting a point on the image $M=(x / z, y / z, 1)^{T}$.

This projective model assumes a perfect lens system with no distortion. Since distortions are non-linear in nature, they cannot be represented in this projective manner. Instead, they are modelled as non-linear functions on the input data and corrected as a pre-processing step before the projective model is applied to the undistorted image.

Two sources of distortion are considered in this paper - radial and tangential distortion.

Radial distortion is caused by imperfections in the lens system. Those are usually the most visible distortions and cause the well known "barrel" or "pincushion" shapes on straight lines in captured images. This kind of distortion is modelled as a quadratic function on the distance from the optical centre with one, two, or even more terms. The radial distortion function is expressed as:

$$
X_{d}=X_{u}\left(1+k_{1} r+k_{2} r+\cdots\right)
$$

where 


$$
r=\left(X_{u}-C\right)
$$

In (3), $X_{u}$ denotes the undistorted point on the image plane, $C$ is the point $\left(c_{x}, c_{y}\right)$ from $K$ and $X_{d}$ is the distorted point observed in the image. Finally, $k_{n}$ are the distortion parameters.

The second type of common distortion is called tangential and is caused when the image sensor is not completely parallel to the optics. It causes trapezoidal shaped distortion and is expressed with the following model:

$$
\left|\begin{array}{l}
x_{d} \\
y_{d}
\end{array}\right|=\left|\begin{array}{l}
x_{u} \\
y_{u}
\end{array}\right|+\left|\begin{array}{l}
\left.d_{2} *\left(r^{2}+2 x_{u}^{2}\right)+2 d_{1} x_{u} y_{u}\right)\left(1+d_{3} r^{2}+d_{4} r^{4}+\cdots\right) \\
\left(d_{1} *\left(r^{2}+2 y_{u}^{2}\right)+2 d_{2} x_{u} y_{u}\right)\left(1+d_{3} r^{2}+d_{4} r^{4}+\cdots\right)
\end{array}\right|
$$

where $d_{n}$ are the tangential distortion parameters. Altogether, $D$ denotes the set of all distortion parameters. From (3) and (5) follows that one can compute where each ray will be projected onto the image plane given the undistorted position:

$$
\left.\left|\begin{array}{l}
x_{d} \\
y_{d}
\end{array}\right|=\left|\begin{array}{l}
x_{u}\left(1+d_{1} r^{2}+d_{2} r^{4}+\cdots\right) \\
y_{u}\left(1+d_{1} r^{2}+d_{2} r^{4}+\cdots\right)
\end{array}\right|+\mid \begin{array}{l}
\left.d_{2} *\left(r^{2}+2 x_{u}^{2}\right)+2 d_{1} x_{u} y_{u}\right)\left(1+d_{3} r^{2}+d_{4} d^{4}+\cdots\right) \\
\left(d_{1} *\left(r^{2}+2 y_{u}^{2}\right)+2 d_{2} x_{u} y_{u}\right)\left(1+d_{3} r^{2}+d_{4} r^{4}+\cdots\right)
\end{array}\right)
$$

Using the inverse of (6), one can take a point from the actual image of the camera and compute its position on an ideal free-from-distortion image plane. There is no easy way of computing the inverse of (6), so close numeric approximations are usually applied in practice.

Finally, there is the position and orientation of the camera according to a fixed world coordinate frame, which is called the extrinsic camera calibration and is described as a 6 degrees-of-freedom (DoF) rigid transformation in $\mathrm{R}^{3}$ consisting of a rotation $R$ expressed as a set of 3 axis angles or a quaternion and a translation vector $t$. We can then use $R$ and $t$ to express the complete projective equation of a distortion free camera as:

$$
p=K[R \mid t] P
$$

\section{B. ESTIMATING THE CAMERA PARAMETERS}

To summarize the previous chapter: one has to estimate 5 parameters of the matrix $K$, between 2 and 8 parameters for the distortion depending on the level of precision needed, and 6 parameters for the position and orientation of the camera for each image taken.

The calibration pattern used for the proposed system consists of 20 April Tags arranged in 4 rows with 5 markers each that can easily be printed on a A4 or A3 sheet of paper and attached to a rigid surface like cardboard or plastic (see Fig. 3).
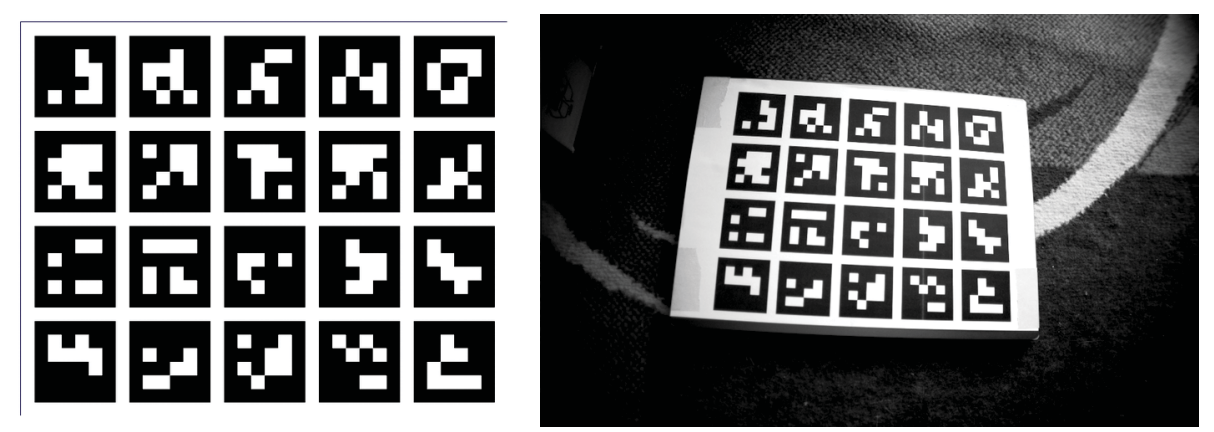

Fig 3: Calibration Pattern.

The user takes pictures of the pattern with the intent to cover as well as possible the image sensor surface while still creating enough motion between the images to avoid degenerate cases where only rotation is applied. After every 5 good images, the calibration process is started and, depending on the quality of the result, a further set of images can be taken or the process completes. After every image is taken, the blur detection algorithm 
as described in [3] is applied. The main goal there is to compare the effect of applying a low pass filter, implemented as artificially introduced directional blur with a large convolution kernel on the image, and compare this to the original image. If the effect of the low pass filter is minimal, then the original image is expected to be blurry as well, while if the effect is high, this means sharp edges in the original image were present. This technique serves the use case very well because many sharp edges are expected in the original image due to the calibration pattern. This method was compared against another metric for blur based on edges detection with the help of the Sobel operator, but it tends to be much less stable and depends heavily on the calibration environment and the size of the calibration pattern in the image.

Every image that does not pass the blur test is excluded from the calibration set to avoid degradation of quality. The images that have passed this test are then fed to the April Tags library for tag extraction. Instead of extracting the centroid of each tag we pick the four corners because this gives more data for the consecutive steps thus making the whole algorithm more robust. Only markers with sufficiently high detection confidence are used and images where less than half the markers are found are removed from the process as well. For an ideal image, this means a grid of $10 \times 8$ points lying on parallel lines is extracted. These points are used to compute a homography matrix between the calibration model plane and the viewing plane of each image. The homography for each image, $H_{i}$, is a $3 \times 3$ matrix defined up to a scaling factor and represents a bijective projective transformation which maps points between two planes. It expresses the mapping between the points on the calibration plane to the points on the image plane of the camera. Due to the distortion of the input images, the projection error of the initial homography matrix is expected to be relatively high, especially in the image corners. The homography estimation is done as described in [8]. Due to the fact that some markers might have still been incorrectly detected in the input image, an approach similar to RANSAC $[4,5]$ is applied.

ALGORITHM 1: Computing the initial Homography $\mathrm{H}_{\mathrm{i}}$

1. Detect all markers $M_{i}$ in the $t^{\text {th }}$ image;

2. Select 2 random markers $m_{a, b} \in M_{i}$;

3. Compute $H_{i}$ as described in [8] from the corners of $m$.

4. Compute the re-projection error $E$ of $H_{i}$ for $\forall m \in M_{i}$.

5. If $E<E_{\text {thresh }}$ then return $\mathrm{H}_{i}$ else goto 2 .

The next step of the calibration process estimates the initial values of the distortion parameters by enforcing the linearity constraint on the edge points of the detected markers. This step is based on the work of Devernay and Faugeras in [7]. This is done is by minimizing the error between a set of "supposed-to-be" lines that are radially distorted, and what they should be, i.e. straight. This is achieved by a non-linear least squares minimization of the re-projection error of the homography $H$ of the undistorted points as defined by the inverse of (6) in the image and their expected position on the ideal lines defined in the calibration model.

At this point, the initial homography $H_{i}$ can be replaced with a refined estimate over the undistorted image points $H_{R i}$. All further steps use $H_{R i}$.

The intrinsic camera parameters $f_{x}, f_{y}, c_{x}, c_{y}$ are extracted from $H_{R i}$ by solving the equations described in sections 2.2 and 2.3 as well as appendix B in [6]. This linear system, given enough images, is overdefined and can be solved by a singular value decomposition of the equation matrix. Also the extrinsic calibration parameters for each image are extracted from $H_{R i}$ by decomposing $H_{R i}$ into a rotation matrix $R_{i}$ and a translation vector $t_{i}$ from the fact that:

$$
H=\lambda K\left|r_{1} r_{2} t\right|
$$


Next, the initial estimates of $R_{i}$ and $t_{i}$ are refined by performing a non-linear least squares minimization of the re-projection error using (7), followed by a non-linear minimization of the intrinsic parameters using all images. At this point, the results should already be very precise. Under time constraints, they can be used as final results because the following steps are more time consuming but can further improve the calibration results.

The last two steps are two bundle adjustments where most parameters are allowed to change during the optimization descend. The first of those optimizes all intrinsic and extrinsic parameters of the cameras in a single non-linear system. The minimization function is:

$$
\sum_{i=1}^{n} \sum_{j=1}^{m}\left\|P\left(\bar{X}_{i j}, K, D, R_{i}, t_{i}\right)-X_{j}\right\|
$$

where $\bar{X}_{i j}$ are the points of the detected markers on the calibration model and $X_{j}$ are the respective points detected in the images.

Finally, the last bundle adjustment step solves another problem which in theory should not exist and is a common source of frustration when doing camera calibration the quality of the calibration pattern. Most users print those on regular sheets of paper and then either directly hold them in front of the camera or glue them onto some surface like cardboard which, though mostly planar, is never perfect and somewhat flexible. Therefore the model points $\bar{X}_{i j}$ of the markers are not necessarily what the real world observed geometry is. In order to take this into account, one last minimization step is performed again using (9) as a minimization function but this time $\bar{X}_{i j}$ is also added to the variable set. It is important to point out that the results of this step should be closely monitored. If large changes in $\bar{X}_{i j}$ are applied, this is an indication that the calibration patter was too bent or the initial estimates of the other parameters are too far off. In this case, the calibration process should be stopped and more image data acquired and a new calibration pattern created if needed.

All steps of the process after computing the initial intrinsic parameter set can be repeated iteratively for further improvements to accuracy. Practical tests have shown that this doesn't usually contribute to measurable improvement in the calibration precision.

The algorithm of the pipeline is presented in Fig. 4.

\section{DEALING WITH STEREO AND MULTI-VIEW SETUPS}

Essentially, multi-view calibration follows the same steps as outlined in Fig. 4. The only moment that requires extra attention is the fact that all rigidly attached cameras must have the same transformation that describes their relative positioning. This can easily be enforced in the extrinsic bundle adjustment step and the complete bundle adjustment.

This paper does not cover the problem of image rectification which is central to stereo vision but the interested reader is advised to see the excellent monograph [10] for extensive discussion on this topic.

\section{EVALUATION}

The results of the calibration framework were compared in terms of quality and efficiency of the calibration process with the tools provided by OpenCV [8] and AprilCal [1].

As the main goal of the proposed system is to provide precise and efficient method for camera calibration which does not require extensive knowledge on the topic, the following test setup has been chosen: a set of three non-trained users were picked and explained the way to use all three applications to calibrate a camera. The explanation was done using the provided materials from the authors and by doing a demonstration of each system. Next the users were asked to complete the calibration of 2 different cameras with each system. One of the cameras was selected to be a low end web camera, as seen in 
most consumer computer systems and a high end device from Point Grey Research - a Grasshopper 3 with Tamron $8 \mathrm{~mm}$ fixed focus and iris lens. Since the different calibration systems require different number of images to complete successful calibration, we decided to settle on 15 images as the maximum number of images a user will take before
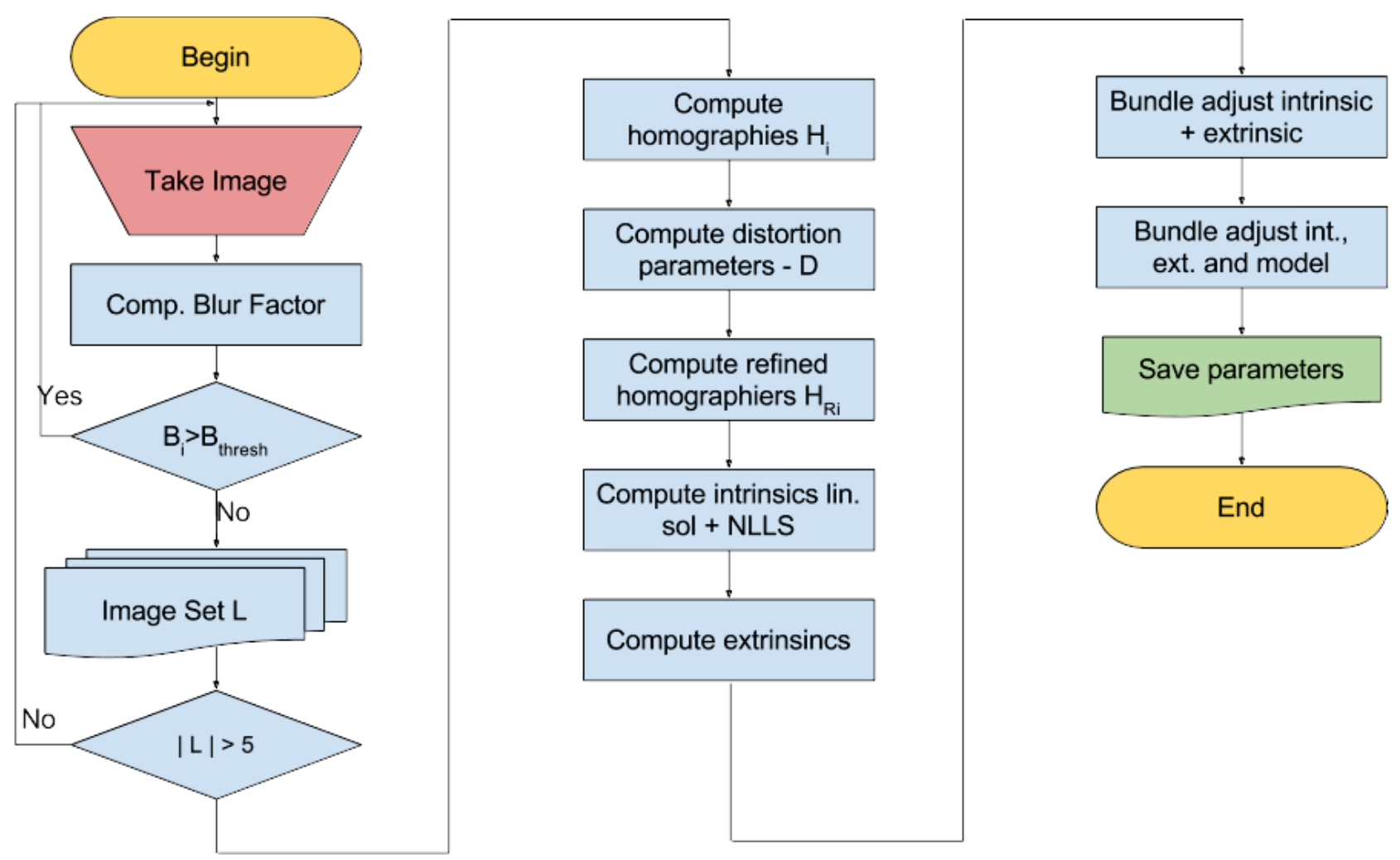

Fig 4: Algorithm of the Calibration Process

requesting results. All systems can benefit equally from increasing this number, but a number was selected that is not overwhelming for an untrained user. The final calibration quality and the time needed to accomplish the task with each system is then measured. To make sure each system is given a fair comparison, each user was given a different order of the systems so that each one has appeared once as first, second, and third system.

The quality was measured as the mean re-projection error of the calibration target in the captured images. The time for calibration was measured from starting the system until the $15^{\text {th }}$ image was taken or the system declared it had finished the calibration process and stopped the process. The average quality results are presented in Table 1 and the averaged times needed to finish the task in Table 2.

\begin{tabular}{|l|r|r|r|} 
Camera & \multicolumn{1}{|c|}{ OpenCV } & \multicolumn{1}{c|}{ AprilCal } & Our system \\
\hline Webcam 640x480 & 0.36 & $\mathbf{0 . 1 7}$ & 0.22 \\
\hline PG GH3 1920x1200 & 0.78 & 0.33 & $\mathbf{0 . 2 7}$ \\
\hline
\end{tabular}

\begin{tabular}{|l|r|r|r|}
\hline Camera & \multicolumn{1}{|c|}{ OpenCV } & \multicolumn{1}{c|}{ AprilCal } & Our system \\
\hline Webcam 640x480 & 312s & 630s & 411s \\
\hline PG GH3 1920x1200 & 357s & 702s & $382 \mathrm{~s}$ \\
\hline
\end{tabular}

Table 1: Calibration Precision in $p x$

Table 2: Calibration Time

The calibration results achieved by the non-professionals were very comparable between the proposed system and AprilCal but the time it took our candidates to try to follow the projected suggestions of AprilCal was more than twice that of what is needed to simply shoot 15 images. The only recommendation that was given for the non-guided shooting for OpenCV and for this system was to try to cover as well as possible the whole surface of the image and in OpenCV's case that it is critical to have the whole pattern visible in all images. It is easily visible that this system provides great solution for nontrained users. It provides optimal compormise of quality and efficiency. 


\section{CONCLUSIONS AND FUTURE WORK}

In this paper, a system for camera calibration was proposed which is capable of producing high quality results while requiring little training of the operator. It combines the benefit of the quick operation of non-guided system, like OpenCV, with the convenience of a calibration target that doesn't have to be completely visible in all images thus, it is easy to cover the image sensor space. Finally it reduces the risk of motion blur affecting the calibration process by measuring it and eliminating bad images from the process.

Future work in this area can be done to provide non-intrusive guidance to the calibration process, similar to AprilCal, but by animating suitable motion trajectories for the operator instead of relying on verbal instructions and projected poses.

\section{REFERENCES}

[1] A. Richardson, J. Strom, E. Olson, Assisted and repeatable camera calibration, Proceedings of the IEEE/RSJ International Conference on Intelligent Robots and Systems IROS, 2013.

[2] F. Crété-Roffet, T. Dolmière, P. Ladret, M. Nicolas, The Blur Effect: Perception and Estimation with a New No-Reference Perceptual Blur Metric, Proceedings of SPIE - The International Society for Optical Engineering, 2007.

[3] Y. Yao, B. Abidi, N. Doggaz, M. Abidi, Evaluation of Sharpness Measures and Search Algorithms for the Auto-Focusing of High Magnification Images, SPIE, 2006.

[4] M.A. Fischler, R.C. Bolles, Random sample consensus: a paradigm for model fitting with applications to image analysis and automated cartography, Communications of the ACM Volume 24 Issue 6, 1981.

[5] P.H.S. Torr, A. Zisserman, MLESAC: A New Robust Estimator with Application to Estimating Image Geometry, Computer Vision and Image Understanding 78(1):138-156, 2000.

[6] Z. Zhang, A flexible new Technique for Camera Calibration, IEEE Transactions on Pattern Analysis and Machine Intelligence, 22(11):1330-1334, 2000.

[7] F. Devernay, O. Faugeras, Straight lines have to be straight: automatic calibration

and removal of distortion from scenes of structured environments, Machine Vision and Applications, 13 (1), pp.14-24, Springer Verlag, 2001.

[8] The Open CV Documentation, Camera Calibration With OpenCV, http://docs.opencv.org/3.1.0/d4/d94/tutorial camera calibration.html.

[9] MathWorks, Estimate Geometric Parameters of a Single Camera, http://mathworks.com/help/vision/ref/cameracalibrator-app.html.

[10] R. Hartley and A. Zisserman, Multiple View Geometry in Computer Vision, Cambridge University Press, ISBN: 0521540518, 2004.

[11] Q. Bao, Blur metric implementation for Matlab, blurMetric.m, 2008.

[12] A. Datta, J. S. Kim, T. Kanade, Accurate camera calibration using iterative refinementof control points, IEEE 12th ICCV, Computer Vision Workshops, 2009.

\section{ABOUT THE AUTHOR}

Yulian Pastarmov, Software Engineer at Google - Munich,Germany; Doctoral Student at the Computer Systems\&Technologies Dep. - St. Cyril and St. Methodius University Veliko Tarnovo, Bulgaria. Phone: +49 176 64105209, E-mail: pastarmovj@google.com. 